\title{
Diacronie
}

Studi di Storia Contemporanea

$N^{\circ} 12,4 \mid 2012$

Sulle tracce delle idee

\section{La costruzione dei discorsi sui cabaret e sulle prostitute durante l'epoca dell'Estado Novo ad Aracaju}

\section{Débora Souza Cruz}

Traduttore: Anita Lucchesi

\section{OpenEdition \\ Journals}

\section{Edizione digitale}

URL: http://journals.openedition.org/diacronie/2491

DOI: 10.4000/diacronie.2491

ISSN: 2038-0925

\section{Editore}

Association culturelle Diacronie

Notizia bibliografica digitale

Débora Souza Cruz, « La costruzione dei discorsi sui cabaret e sulle prostitute durante l'epoca dell'Estado Novo ad Aracaju », Diacronie [Online], № 12, 4 | 2012, documento 4, Messo online il 29 décembre 2012, consultato il 11 juin 2020. URL : http://journals.openedition.org/diacronie/2491 ; DOI : https://doi.org/10.4000/diacronie.2491 


\section{Diacronie}

\section{La costruzione dei discorsi sui cabaret e sulle prostitute durante l'epoca dell'Estado Novo ad Aracaju}

\section{Débora SOUZA CRUZ *}

traduzione di Anita LUCCHESI

Questo articolo si propone di analizzare i discorsi medici e giornalistici costruiti intorno alle figure delle prostitute ed ai cabaret durante il periodo dell'Estado Novo (1937-1945) nella città di Aracaju. L'Estado Novo si caratterizzò come uno dei periodi più autoritari della storia del Brasile, durante il quale Getúlio Vargas cercò di controllare la popolazione brasiliana. Come strategia per applicare le sue misure di governo furono nominati alcuni interventores. Uno dei suoi obiettivi principali era conseguire la modernizzazione, la civilizzazione e l'igiene dei corpi, contando principalmente sull'appoggio dei medici dell'epoca. Considerati come ostacoli a tale progetto di modernizzazione, le prostitute ed i cabaret erano spesso presi di mira dalla stampa locale e venivano descritti negativamente. Tuttavia, nonostante la vigilanza e tutti gli stereotipi prodotti su queste donne e su questi luoghi, essi non hanno mai cessato di esistere, ma sono giunti ad essere considerati come un "male necessario".

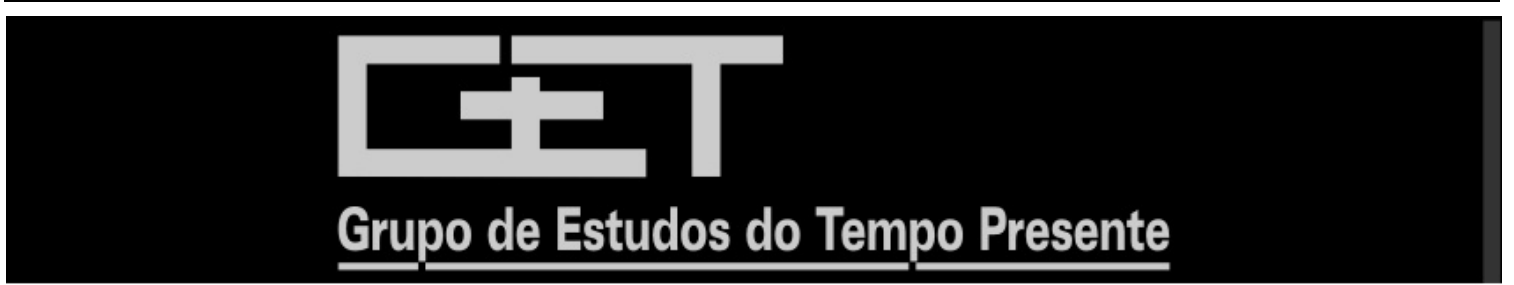

Il presente articolo viene pubblicato in traduzione italiana per gentile concessione della rivista Cadernos do Tempo Presente nell'ambito del progetto di collaborazione tra le due riviste. 


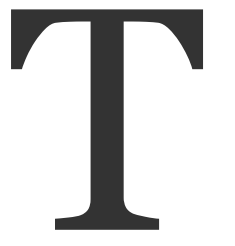

ra il 1937 e il 1945, il Brasile ha vissuto uno dei periodi più autoritari della sua storia. L'Estado Novo si è sviluppato in Brasile nel periodo tra le due guerre, durante il quale il sistema liberale versava in crisi a causa dell’incapacità di risolvere i problemi sociali. Di fronte a questa situazione e alle critiche rivolte alla democrazia, una delle soluzioni trovate fu quella di realizzare uno Stato centralizzatore, guidato da un forte e carismatico leader, in grado di stabilire l'ordine tra le masse. Così, Getúlio Vargas divenne il leader del paese e guidò uno dei regimi più repressivi della storia nazionale. Grazie a una forte concentrazione di potere nell'Esecutivo Federale, alla censura attraverso il DIP (Dipartimento di Stampa e Propaganda) e al divieto ufficiale di espressione di qualsiasi opinione contraria al suo governo, Vargas cercò di controllare la vita quotidiana della popolazione. Nello stato del Sergipe, il primo interventor ${ }^{1}$ di questo periodo fu il medico Eronides de Carvalho².

Tra gli ambienti che dovevano essere maggiormente controllati e sorvegliati a quel tempo c'erano i cabaret. Questi, secondo il governo, riunivano i tre elementi che avrebbero dovuto essere evitati, soprattutto dagli uomini aracajuniani: alcool, orge e gioco d'azzardo. Le prostitute e i loro ambienti di lavoro hanno rappresentato un ostacolo alla modernizzazione a al disciplinamento che Vargas cercava di conseguire con il suo governo.

Preoccupati dalla presenza delle prostitute e dalla loro frequente richiesta da parte degli uomini e dei ragazzi aracajuniani, il governo, i medici e gli operatori sanitari, informarono la popolazione di Aracaju sull'importanza dell'educazione morale e sessuale, che i genitori avrebbero dovuto trasmettere ai loro figli, soprattutto quelli maschi. È importante ricordare che in questo periodo - gli anni Trenta e Quaranta - la conoscenza medica era considerata la sola legittima e, inoltre, i professionisti in campo sanitario si occupavano di trasformare le città in spazi civilizzati e liberi dalle malattie e dalla scarsa igiene; tuttavia, tale compito non era facile.

\footnotetext{
${ }^{1}$ Gli interventores erano uomini di fiducia del Presidente Vargas, nominati per ciascuno Stato per occupare la funzione che era in precedenza svolta dai governatori; questa figura fu istituita a partire dal 1930, a seguito della Rivoluzione.

${ }_{2}^{2}$ Eronides Ferreira de Carvalho nacque a Canhoba (Sergipe), il 25 aprile 1895. Nel 1911 si iscrisse alla Facoltà di Medicina di Bahia, concludendo il percorso di studi nel 1918. L'anno successivo, divenne direttore ad interim della Sanità pubblica dello Stato del Sergipe; nel 1923, fu promosso nella divisione medica dell esercito. Nel 1924, divenne primo tenente e due anni dopo fu assegnato al gruppo che diede la caccia alla "Colonna Prestes" - un movimento politico militare legato al tenentismo - nel Nord-Est. Nel 1935, eletto indirettamente, divenne governatore costituzionale. Morì nella città di Rio de Janeiro, dove diresse un studio notarile, nel marzo 1969. Su Dom José Tomaz si veda: BARRETO, Luiz Antonio, Dicionário de nomes de denominações de Aracaju, Aracaju, Banese, 2002.
} 
Esempio della difficoltà di far mettere in pratica i nuovi comportamenti alla popolazione aracajuana, è una nota di sfogo scritta da un reporter nel «Jornal Folha da Manhã». Il giornalista sedeva in una delle caffetterie della città, il Café Moca all'incrocio tra rua João Pessoa e rua Laranjeiras -, quando vide un «uomo del popolo» che si avviava al fiume Cotinguiba per gettare lì un cavallo che versava già in stato di putrefazione. La riprovazione del reporter si esprimeva così: «tale abitudine, propria di persone prive di istruzione, ha bisogno di un correttivo, soprattutto ora che il nostro servizio sanitario pubblico è così severo ed efficace»3. Nelle ultime parole del reporter, ci rendiamo conto di come questo fosse un periodo in cui si stava seriamente cercando di introdurre i cambiamenti in materia di politica sanitaria nella capitale dello Stato del Sergipe.

Tutta questa preoccupazione da parte del giornalista nei confronti delle "cattive abitudini" ancora presenti nella capitale, era da mettere in relazione con il desiderio che Aracaju fosse collocata nel contesto della modernizzazione e della civilizzazione, soprattutto prendendo spunto dalle città europee. Per questa ragione, non solo il giornalista, ma anche il governo aspirava a trasformare alcune abitudini della popolazione. Questi cambiamenti erano legati alle abitudini in materia di sanità pubblica, di servizi igienico-sanitari e di pulizia dei corpi: infatti proprio la mancanza di igiene e la richiesta di prostitute rappresentavano un ostacolo alle misure morali e progressiste, non solo per Aracaju, ma per l'intera nazione.

Oltre ai medici e agli operatori sanitari, la stampa locale svolse il ruolo di divulgatrice dei valori del progetto civilizzatore, ma al contempo riuscì efficacemente anche ad attribuire qualità dispregiative e cliché a tutto ciò che riguardava le "donne di facili costumi”. Spesso, diversi discorsi erano costruiti e divulgati attraverso giornali e riviste: qui le prostitute ed i cabaret erano i principali obiettivi degli attacchi. Considerando l'affermazione di Foucault, secondo cui «ogni sistema di educazione è un modo politico di mantenere o di modificare l'appropriazione dei discorsi»4, emerge come fu proprio attraverso i discorsi pubblicati dalla stampa che Vargas cercò di conservare la morale e le buone pratiche nella capitale, come si può osservare da un trafiletto intitolato «La cattiva educazione, fonte di cattiva salute fisica e morale»5:

[...] Quando il ragazzo è di fronte alle difficoltà e ai bisogni sociali cerca un mezzo per fuggirne. Questo mezzo può essere rappresentato dalle sostanze tossiche, dall'alcool, dalle

\footnotetext{
3 «Um péssimo hábito que precisaria desaparecer», in Folha da manhã, 10 gennaio 1939, p. 1. 4 FOUCAULT, Michel, L'ordine del discorso, Torino, Einaudi, 1970, pp. 22-23.

5 LIMA, Hermes, «A má educação, fonte de má saúde física e moral», in Folha da manhã , 14 aprile 1939.
} 
perversioni sessuali, se nessun profondo legame emotivo o un'irrefrenabile inclinazione religiosa non lo salva da queste miserie estreme $[\ldots]^{6}$.

Durante questo periodo, la religione e la famiglia furono considerate come due istituzioni solide e sicure per far sì che gli uomini non cercassero una casa di tolleranza, i casinò o i bar come sfogo per le loro perversioni sessuali. Se la strada intrapresa che veniva imboccata era quella dei cabaret, si rischiava che il matrimonio - che veniva fortemente incentivato dalla Chiesa - potesse incrinarsi, avviando un divorzio. Anche il divorzio non era visto con favore dai "moralisti" del tempo perché, a loro giudizio, la società avrebbe marciato "verso la dissolutezza, sviando i sentimenti da ciò che più deve essere rispettato, la famiglia» 7 . Inoltre, era un uomo perbene e rispettabile quello che viveva con una sola compagna fino all'ultimo giorno della sua vita: solo in questo modo si sarebbe potuta scongiurare la prostituzione.

Il fenomeno della prostituzione si produsse in una società profondamente moralista e caratterizzata dall'apprezzamento di quei valori espressi dalla Chiesa: l'unione sessuale monogama, la famiglia e la verginità. Così, ancora prima dei discorsi e delle critiche sollevate durante il periodo dell'Estado Novo sulla prostituzione, la sua immagine si era già connotata negativamente.

Anche senza considerare il quadro dei valori difesi dalla Chiesa e dalla società, le prostitute occupavano una posizione completamente opposta rispetto a quella della "regina della casa", della madre esemplare. Le meretrici, che avevano scelto di vivere sessualmente libere, nella maggior parte dei casi non erano disposte a dedicare la loro vita al matrimonio e alla maternità, considerate le due principali funzioni che dovevano essere svolte dalle "donne normali": tutto ciò non faceva che aumentare ulteriormente il pregiudizio su di loro; malgrado ciò, non sempre è possibile tratteggiare quel confine prevedibile tra "regina della casa" e "signora della notte".

Nel suo Aracaju romântica que vi e vivi, il memorialista Melins Murillo racconta che una notte, recatosi a un appuntamento in un bar nel centro della città, sentì la voce di un bohémien accanto al suo amico chiedere chi fossero quelle donne alla moda che scrutavano le vetrine dei grandi magazzini. Si sentì rispondere che si trattava di "donne della notte" che uscivano da pensioni come la "Mira-mar" e venivano, così come qualsiasi altra donna, ad apprezzare i vestiti della «fiera della vanità» 8 .

\footnotetext{
${ }^{6}$ Ibidem.

7 MELO, Luiz Pereira de, «O divórcio», in Folha da manhã, 5 febbraio 1939, p. 5.

8 MELINS, Murillo, Aracaju Romântica que vi e vivi, Aracaju, Unit, 2007. p. 179.
} 
Anche la società tentava al massimo grado di imporre una separazione ed una differenzazione tra le donne di facili costumi e le donne "rette", poiché queste non sempre vestivano con abiti ed accessori che potessero farle identificare come tali. Molte prostitute di lusso frequentavano luoghi in cui si trovavano anche le donne rispettabili9.

Malgrado questa distinzione non fosse sempre evidente, era necessario considerare le donne di facili costumi come persone che deviavano dal cammino della morale che doveva essere seguita; così, divenne importante mostrarle alla società come la causa principale della disgregazione delle famiglie, in aggiunta alle altre immagini negative che erano affibbiate quotidianamente alle meretrici.

Un'altra rappresentazione spesso rinvenibile era quella di essere responsabili della diffusione della sifilide e di altre malattie veneree. Questo discorso fu sostenuto principalmente da medici e specialisti che cercavano costantemente di contenere l'aumento delle malattie sessualmente trasmissibili e motivavano le origini di queste con il crescente commercio dei corpi, come emerge dai seguenti brani tratti dal «Jornal Folha da Manhã»:

[...] A prima vista sembra che il problema delle malattie veneree non abbia anche le sue origini nel pauperismo, se non per una mancanza di educazione sanitaria. Pensiamo al contrario. Cominceremo dal problema della prostituzione, unico fattore della trasmissione delle malattie veneree [...] la prostituzione è una macchia nella società di una nazione, mercato di carne per il sostentamento di infelici creature $[\ldots]^{10}$.

Nel breve articolo medico sopra riportato percepiamo ancora una volta la preoccupazione di investire nelle comunicazione dirette alla popolazione aracajuniana su temi riguardanti l'educazione sessuale e sanitaria, dal momento che, secondo il testo, il problema delle malattie veneree non era legato allo stato finanziario della città, quanto, piuttosto, alla mancanza di prescrizioni igieniche. Fu proprio considerando questo problema che il governo, insieme ai professionisti nel campo della sanità, realizzò il 2 ottobre 1945, la prima "Settimana Anti-Venerea" nella capitale dello Stato del Sergipe ${ }^{11}$.

\footnotetext{
9 RAGO, Margareth, Os prazeres da noite: prostituição e códigos da sexualidade feminina em São Paulo (189o-1930), São Paulo, Paz e Terra, 2008, p. 37.

10 «Nota medica» in Folha da manhã, 22 settembre 1945, p. 2.

11 «Primeira Semana Anti-Venérea», in Folha da manhã, 28 settembre 1945, p. 2.
} 
Il programma prevedeva discorsi pubblici, conferenze e interventi che furono realizzati presso l'Istituto Storico e Geografico di Sergipe, oltre che nelle fabbriche, nei licei e presso le corporazioni militari. Gli interventi contarono principalmente sulla presenza di medici del Dipartimento di Sanità come il dottor Carlos Firpo ${ }^{12}$, il dottor Carlos Souto e il dottor Walter Cardoso, allora direttore del Dipartimento di Sanità.

$\mathrm{Al}$ di là della necessità di realizzare una prima "Settimana Anti-Venerea", notiamo che le ricerche fatte dai medici e dagli igienisti non erano orientate a beneficio delle prostitute, ma all'opposto, servivano per stereotiparle ancora di più. Le meretrici erano descritte como «infelici creature» che costituivano, per via del loro lavoro, una «macchia» non solo per la città di Aracaju, ma per tutta la nazione: erano loro, con il commercio dei loro corpi che lasciavano una «sozzura» per le strade, oltraggiando i valori morali ed igienici che si cercava di introdurre. Erano loro a contribuire alla diffusione delle malattie.

Questi principi igienici e morali erano accompagnati da una nuova politica sanitaria che si stava imponendo non solo ad Aracaju, ma in luoghi diversi del Brasile fin dal 1920 ${ }^{13}$. Tuttavia, dobbiamo prestare attenzione al fatto che le prostitute, pur identificate come la principale forma di infezione della sifilide, non erano quasi mai oggetto degli investimenti nell'educazione sessuale e nella consulenza sanitaria. La conseguenza di ciò era che esse finivano per divenire ancora più vulnerabili alle malattie veneree e venivano considerate come immonde, brutte e malate ${ }^{14}$.

È con queste parole sopra riportate che il memorialista Mário Cabral inquadra nella sua opera, Rotta di Aracaju, le signore della notte che vendevano i loro corpi nel Curral. A suo parere si trattava di «donne brutte, magre, coperte di stracci, consumate dalle malattie più gravi e vergognose, che vendevano per un piatto di cibo il loro corpo malato, in una parvenza d'amore che provocava nausea e pietà ${ }^{15}$. Tali caratteristiche trasfiguravano, diffondevano e anche generalizzavano un modello di meretrice che non risultava minimamente invitante per gli uomini.

Non diversamente dal memorialista Cabral, altri poeti del tempo riportavano nelle loro opere - che venivano pubblicate sui giornali e sulle riviste della capitale caratteristiche negative sulle lucciole. Una di queste poesie intitolata Al Cabaret (No

${ }^{12}$ Carlos Firpo era considerato un medico di fama, oltre ad aver guidato il Comune della città di Aracaju tra gli anni 1941 e 1942. Il 23 marzo 1958, Firpo fu ucciso in casa sua durante il sonno. Questo crimine fu all'epoca oggetto di molti commenti e scosse la città di Aracaju. Sull'argomento si veda BARRETO, Luiz Antonio, Dicionário de nomes de denominações de Aracaju, Aracaju, Banese, 2002.

13 MAYNARD, Dilton, Cafés e pinga-pus na Aracaju da II Guerra (1939-1945), Universidade Federal de Sergipe, Relatório final PIBIC, 1998, p. 3.

14 RAGO, Margareth, op. cit., p. 162.

15 CABRAL, Mário, Roteiro de Aracaju, Aracaju, Banes, 2001, p. 124. 
Cabarét), venne stata pubblicata sulla rivista «Novidade», con la firma di José dos Anjos Fábio:

\author{
Vagando attraverso una fredda notte d’inverno, \\ Sento rumori, gazzarra, un inferno \\ Risvegliando in me una strana gioia [...] \\ I miei occhi vogliono vedere [...] Mi insinuo, infine, nella casa dell'orgia. \\ Avversando quello che c'è di puro e cast[r]o, \\ Donne febbrili si contorcono seminude [...] \\ [...] Una donna magra, di pallida bellezza \\ Fuma, sorride, beve e canta come matta! \\ $[\ldots .$.$] Una risata di scherno le sfugge dalla bocca [... { }^{16}$.
}

Nei versi precedenti si nota ancora una volta la presenza degli stereotipi negativi costruiti intorno alle donne "di facili costumi". Anche in questo caso si presentano come malate, scheletriche, non presentabili e dedite ai vizi che dovrebbero essere evitati: alcool e orge. Tuttavia, nonostante questa realtà sia ben sottolineata dal poeta, è da notare come si scateni un interesse e una curiosità anche in lui, appena ode i rumori e la "gazzarra" provenienti dall'interno del cabaret.

Anche se il mondo delle meretrici si presentava terribile, risvegliava nell'autore «una strana allegria»17 che lo invita ad entrare nel bordello. Era proprio per costruire un ambiente così spaventoso, ma allo stesso tempo così affascinante, che questi spazi si costituivano e dovevano essere descritti alla popolazione come cupi ${ }^{18} \mathrm{e}$ pericolosi: locali che erano utili solo all'infezione da malattie sessualmente trasmissibili.

Era sulla base di questa descrizione, ossia, di un luogo che non doveva essere frequentato perché poteva portare soltanto all'infezione, che gli annunci pubblicitari di unguenti, elisir e medici specialisti garantivano la guarigione dalla sifilide in pochi giorni. Come possiamo notare dai manifesti che seguono, sia il cabaret che le meretrici, erano interpretati come "una porta aperta a molti mali» e bastava il contatto con entrambi, sia con la "donna orizzontale", che con i bordelli, perché queste «malattie proprie dell'indole cattiva» fossero facilmente contratte.

\footnotetext{
${ }^{16}$ ANJOS, José Fábio dos, «No Cabarét», in Revista Novidade, 4, 14, 1939.

17 Ibidem.

18 POZZOLI, Marilita, «No cabaret», in Correio de Aracaju, 28 settembre 1945, p. 4.
} 


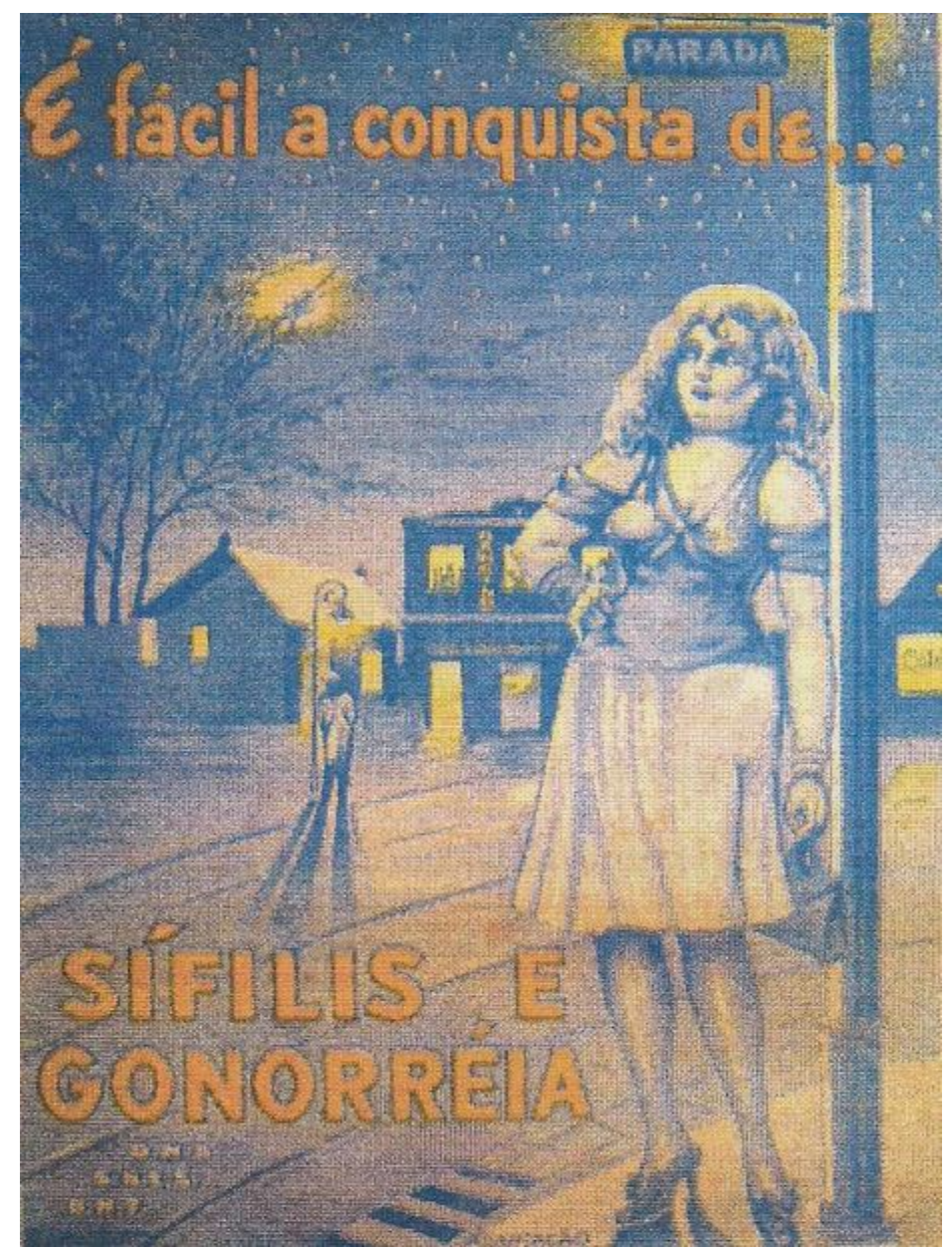

Fig. 1 - Poster della campagna anti-venerea del Ministero della Educazione e Salute di 1930 e 1940. Raccolta IHGS.

Nella figura 1 possiamo vedere raffigurati nella stampa questi mali accostati alla meretrice ed al suo posto di lavoro. La prostituta è in un ambiente buio, nel quale la luna e le stelle sono presenti in cielo, il che può essere collegato al suo turno di lavoro o anche all'atmosfera cupa ed al male che viene attribuito ai cabaret. Gli alberi, che sono vicini al postribolo, non hanno alcun frutto e sono rinsecchiti, a comprovare ancor più come questo sia un ambiente triste e "senza vita".

La prostituta si trova appoggiata a un palo, che funge anche da fermata per i mezzi pubblici, ma che in quel momento invita gli uomini aracajuani a fermarsi e a conoscere la meretrice. Un altro elemento che fa sì che i bohémiens non resistano alla prostituta è la sensualità della stessa, dato che si presenta con una posa e con vestiti abbastanza provocatori per quell'epoca (gonna, tacchi alti, abito che lascia le spalle scoperte). Si nota anche la vicinanza della prostituta alla fogna, a riprova di come sia il cabaret, sia la prostituta, si trovino in un ambiente sudicio e decadente, da cui le nozioni di igiene e 
modernizzazione rimangono assai lontane. Ancora nella figura 1 notiamo la porta aperta del cabaret, a facilitare l'ulteriore contagio dalle malattie veneree.

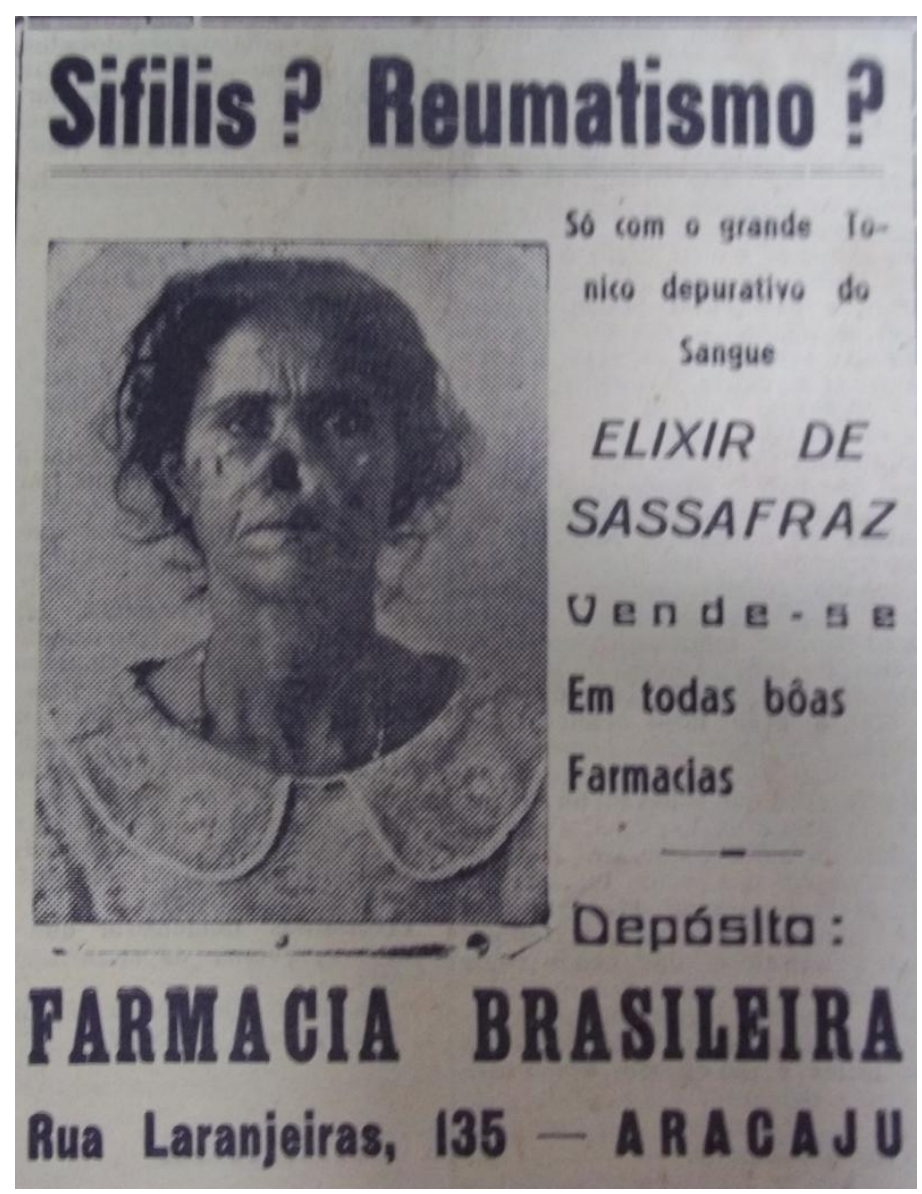

Fig. 2 - Pubblicità dell'elisir di Sassafraz. Correio de Aracaju, 26 gennaio 1938, p. 3.

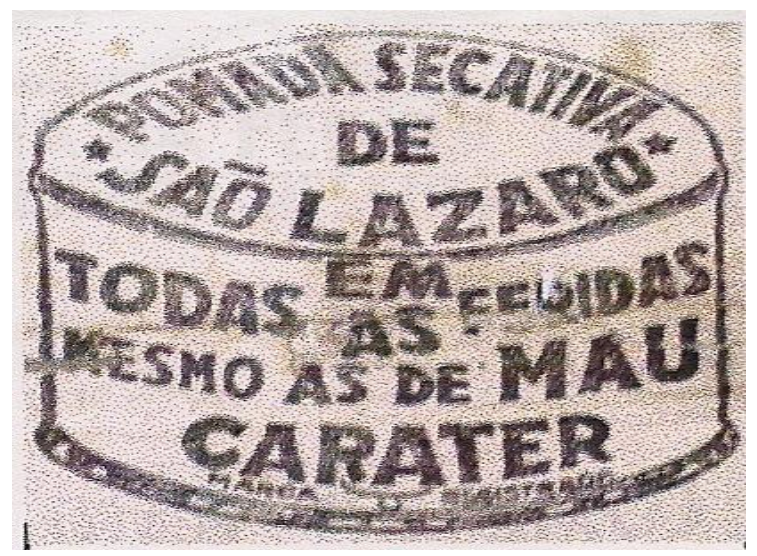

Fig. 3 - Pubblicità della pomata di São Lazaro [parziale].

Correio de Aracaju, 26 maggio 1938, p. 3. 


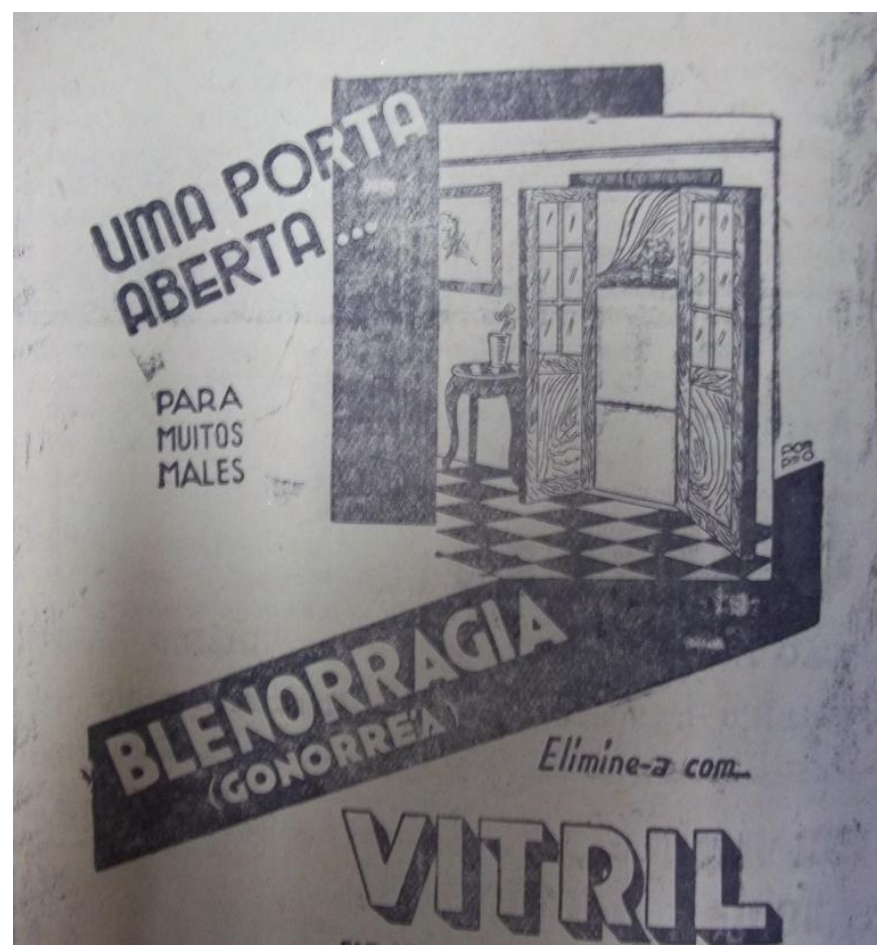

Figura 4 - Pubblicità dell'elisir Vitril [parziale].

Correio de Aracaju, 26 febbraio 1938, p. 2.

Questa immagine della "porta aperta" è presente anche nella figura 4, dove rappresenta un percorso «per molti mali». Mali che non si limitavano alle malattie veneree, ma che si scontravano con ogni concetto di moralità sostenuto dalla Chiesa. Ł̀ proprio per via della «mancanza di buona condotta e di indole» da parte degli aracajuani che frequentavano i cabaret, che la figura 3, pubblicità dell'unguento di San Lazzaro, promette la cura per «tutte le ferite, anche la cattiva inclinazione».

Oltre alla caratterizzazione dei bordelli come ambienti oscuri, propri di donne scheletriche, pallide, malate e brutte, i cabaret erano rappresentati dalla stampa anche come un palcoscenico per le scene di violenza e di tumulto. Il 13 maggio 1938, il giornale «O Nordeste» denunciava il Brahma Bar, dove erano presenti molte lucciole dopo le ore 22:

[...] il Brahma incrostato com'è, costituisce una macchia nera nel cuore di Sergipe, di cui i principi di civiltà non consentono il mantenimento in essere. Brahma, oltre ad essere un insulto alla memoria di João Pessoa, è un oltraggio alla società, dato la sua dubbia frequenza. [...] Il Brahma, di cabaret, anche se di $5^{\text {a }}$ categoria, ha solo il libertinaggio, la dissolutezza, il vizio e l'irresponsabilità [...] collocato così, frequentato com'è; facendo risuonare nelle orecchie di coloro che passano 
attraverso le sue porte il complesso di urli sterici di donne portatrici di facili piaceri $[\ldots]^{19}$.

Anche in questo caso l'espressione «macchia», sinonimo di cattiva fama e caricata di un'accezione negativa in riferimento ai bordelli, è utilizzata nei discorsi presenti sui giornali locali. In questo caso, uno dei maggiori biasimi dell'autore è che l'indirizzo a cui si trovava il bar, rua João Pessoa ${ }^{20}$ - nel centro della capitale - oltre a non rendere giustizia e onore al famoso politico a cui era intitolata la via, non avrebbe dovuto trovarsi in una posizione così centrale, dal momento che la strada era molto affollata a causa del gran numero di negozi.

Sempre in merito alla posizione del Bar Brahma, dobbiamo sottolineare che al di là di questo bar, che era anche un bordello, tanti altri cabaret si concentravano nelle zone centrali e commerciali della città e ne facevano un punto di forza per attirare i clienti appartenenti a diverse classi sociali. Il breve articolo precisa anche come le prostitute lì presenti fossero turbolente o squilibrate, contribuendo così al caos della città e minacciando la tranquillità dei residenti. Seguendo la stessa caratterizzazione costruita per le prostitute e per i cabaret, ancora una volta il Bar Brahma appare nella stampa come un ambiente favorevole ai disordini.

Un venerdì, più precisamente l'8 ottobre 1937, un violento fatto di sangue ebbe luogo nel bar. La contesa ebbe luogo a tarda notte, tra José Vianna e un «uomo di bordo», conosciuto come il Conte. L'elemento che maggiormente richiama la nostra attenzione è che l'incidente fu causato dalla gelosia che Vianna provava per una prostituta «di sua predilezione», chiamata Maria Augusta, che si trovava lì, nel locale, intenta a scambiare effusioni con il marinaio.

Dopo uno scambio di tenerezze al bar, il Conte decise di valersi delle prestazioni della "donna orizzontale" nella casa di questa stessa, ma prima di dirigersi verso l'abitazione di Maria Augusta, che si trovava tra rua Laranjeiras e rua Siriri, il marinaio si recò sulla sua nave e prese la sua arma di fuoco; nel frattempo, Vianna, sconsolato e ancora sotto l'effetto dell'alcool, si diresse a sua volta verso la casa di Augusta. Quando i due si incontrarono nuovamente, sorsero ulteriori discussioni che si conclusero con quattro colpi di pistola, esplosi dal marinaio in direzione di Vianna.

Pur essendo a conoscenza del fatto che la morte era stata causata dal marinaio soprannominato il Conte, il principale responsabile per l'evento, secondo il giornale,

19 «A Brahma», O Nordeste, 13 maggio 1938, p. 1.

${ }^{20}$ João Pessoa Cavalcanti de Albuquerque nacque a Recife nel 1878 e morì nel 1930 nella sua città natale, dove venne ucciso. Acquisì la sua fama in politica, dove ricoprì, tra le altre, la carica di governatore dello Stato di Paraíba. Si veda BARRETO, Luiz Antonio, op. cit. 
risultava la prostituta Maria Augusta, considerata quale «oggetto della contesa». Alla fine del reportage su quanto avvenuto leggiamo che «Maria Augusta fu portata in carcere e al cadavere dell'infelice fu data sepoltura $»^{21}$. L'assassino non venne arrestato e neppure menzionato dal quotidiano. Vianna, che si era diretto a casa della prostituta e aveva scelto di dar seguito al litigio, finiva per risultare una vittima. Al posto del marinaio, Maria Augusta - che svolgeva una professione basata sul commercio del suo corpo e non incorreva quindi nell'obbligo di giacere con un solo uomo - venne arrestata e considerata come la principale causa dell'accaduto.

Responsabili della rovina delle famiglie, «oggetto» di contese che si concludono con la morte, propagatrici della sifilide, opposte alla figura di donna-moglie-madre, brutte, malate e scheletriche, abitanti in ambienti sporchi: malgrado tutti questi stereotipi costruiti intorno alla figura delle meretrici e tutta la sorveglianza dedicata alle case di tolleranza durante l'Estado Novo, la prostituzione, i suoi personaggi e i suoi ambienti non cessarono di esistere. Alcuni motivi sono alla base della permanenza dell'amore venale.

Una ragione possibile è che, nonostante le donne di facili costumi non fossero viste con favore dalla maggioranza degli aracajuani, abbia giocato un ruolo fondamentale il fatto che queste avviassero alla vita sessuale i ragazzi di Aracaju. Generalmente l'inizio della vita sessuale dell'adolescente si verificava in un bordello e, non di rado, il ragazzo veniva portato lì da suo padre o da un parente stretto. Proprio perché il primo rapporto sessuale avveniva con una "donna pubblica", il giovane sarebbe stato capace di liberare i suoi impulsi libidinosi nelle case di tolleranza, mantenendo così le "ragazze rette" e le future spose vergini. In questo modo la prostituzione poteva essere accettata come un male necessario. Se da una parte questo atteggiamento era moralmente condannato dalla società, dall'altra era benaccetto perché rappresentava una garanzia per il mantenimento di alcuni valori e comportamenti come, ad esempio, la verginità.

Così come i ragazzi avevano il loro primo rapporto sessuale con le prostitute e si sbarazzavano del "peccato carnale", molti uomini sposati mantenevano rapporti con prostitute con la scusa che la moglie non poteva soddisfare i loro feticismi sessuali, in quanto questi erano ritenuti anormali e perversi per le donne "rette". Le donne considerate come normali non potevano rivelare la loro sessualità naturale, perché rischiavano di essere paragonate alle "donne di vita”. Inoltre, era nel cabaret che ogni cliente perdeva la propria identità durante qualche ora di piacere «per compensare la

${ }^{21}$ «Violenta scena de sangue!» in Correio de Aracaju, 8 ottobre 1945, p. 4. 
monotonia della vita familiare»22. Mentre la castità delle loro mogli veniva mantenuta, le prostitute rappresentavano un "menu differente" per i propri clienti ed entravano in scena interpretando i personaggi e i desideri richiesti dai loro clienti.

Giocando il ruolo di iniziatrici sessuali e preservando la verginità e la castità delle ragazze e delle madri di famiglia, i bordelli non rappresentavano soltanto un ambiente favorevole per il piacere. Una visita ad un bordello non sempre rappresentava la ricerca di sesso: molti dei clienti vi giungevano alla ricerca di buona musica da ascoltare o di un momento di chiacchiere con i propri compagni di scorribande, stabilivano contatti politici, discutevano affari, scrivevano poesie e, al contempo, qui traevano ispirazione per le future composizioni letterarie, anche se queste finivano poi per contribuire alla cattiva fama della meretrice o del bordello. Non solo: «era possibile addirittura iniziare una carriera artistica. Alcuni degli artisti della Radio sergipana avevano avuto i loro primi contratti presso strutture come queste» ${ }^{23}$. Oltre a rappresentare un ambiente di socializzazione, non dobbiamo dimenticare che negli anni Trenta e Quaranta, la città di Aracaju non aveva molti luoghi di intrattenimento.

I cabaret erano luoghi in cui il tempo libero e il piacere potevano essere goduti a un prezzo accessibile. La maggior parte dei lavoratori, ad esempio, non aveva soldi da spendere negli spazi raffinati come il Café Ponto Chic. Pertanto, tra le scelte più gettonate, ricadevano il Vaticano, il Bomfim, il Mira-Mar e tanti altri locali a luci rosse.

Dopo una giornata dedicata al lavoro gli operai approfittavano della vita notturna e si ritempravano dal lavoro e dalla disciplina industriale. In un periodo storico segnato dalla censura, dalla sorveglianza e dalla repressione come quello dell'Estado Novo di Vargas, la costruzione di queste reti sociali e la ricerca di questi ambienti dimostrava che le persone cercavano momenti di distrazione e libertà, anche se per un breve lasso di tempo. Il mondo del piacere favoriva l'avvicinamento dei singoli individui al collettivo, il che finiva per mettere in secondo piano tutte le caratterizzazioni negative delle donne di facili costumi e dei cabaret, che finivano per essere considerati più come un peccato che come una malattia.

22 RAGO, Margareth, op. cit.

23 MAYNARD, Dilton, op. cit., p. 6. 


\section{Riferimenti bibliografici}

BARRETO, Luiz Antonio, Dicionário de nomes de denominações de Aracaju, Aracaju: Banese, 2002.

BASSERMANN, Lujo, História da prostituição: uma interpretação cultural, Rio de Janeiro, Civilização Brasileira, 1968.

BUSSETO, Áureo, «Em busca da caixa mágica: o Estado Novo e a televisão» in Revista Brasileira de História, 27, 54, 2/2007, URL:

$<$ http://www.scielo.br/scielo.php?pid=S0102-01882007000200010\&script=sci_arttext $>$.

CHALHOUB, Sidney, Trabalho, Lar e Botequim: o cotidiano dos trabalhadores no Rio de Janeiro da belle époque, São Paulo, Editora da UNICAMP, 2001.

DANTAS, Ibarê, História de Sergipe: República (1889-20oo), Rio de Janeiro, Tempo Brasileiro, 2004.

DANTAS, Ibarê, Os partidos políticos em Sergipe (1889-1964), Rio de Janeiro, Tempo Brasileiro, 1989.

DOIN, José Evaldo de Mello, NETO, Humberto Perinelli, PAZIANI, Rodrigo Ribeiro, PACATO, Fábio Augusto, «A Belle Époque caipira: problematização e oportunidades interpretativas da modernidade e urbanização no mundo do Café (1852-1930)» in Revista Brasileira de História, 27, 53, 1/2007, URL:

<http://www.scielo.br/scielo.php?pid=S0102-01882007000100005\&script=sci_arttext>.

ENGEL, Magali, Meretrizes e doutores: saber médico e prostituição no Rio de Janeiro (1840-189o), São Paulo, Brasiliense, 1989.

FONSCECA, Silva, Getúlio Vargas, São Paulo, O Globo, 2004.

FOUCAULT, Michel, L'ordine del discorso, Torino, Einaudi, 1970.

MATOS, Maria Izilda Santos de, Cotidiano e Cultura: História, Cidade e Trabalho, São Paulo, EDUSC, 2002. 
MAYNARD, Dilton Cândido Santos, A arma retórica: o uso do rádio em Sergipe (1939-1945), São Cristóvão, UFS, 2003.

MAYNARD, Dilton Cândido Santos, Em tempos de guerra: aspectos do cotidiano em Aracaju durante a Segunda Guerra Mundial (1939-1945), São Cristóvão, UFS, 1998.

MAYNARD, Dilton, «A redenção da raça: apologia corporal na Aracaju dos annos 30 e 40», in Revista de Aracaju, 1, 11, 2005 pp. 87-102.

NASCIMENTO, Uelba Alexandre de, O doce veneno da noite: prostituição e cotidiano em Campina Grande (1930-1950), Campina Grande, EDUFCG, 2008.

PESAVENTO, Sandra Jatahy, «Cidades visíveis, cidades sensíveis, cidades imaginárias», in Revista Brasileira de História, 27, 53, 1/2007, URL:

<http://www.scielo.br/scielo.php?pid=S0102-01882007000100002\&script=sci_arttext $>$.

RAGO, Margareth, Do cabaré ao lar: a utopia da cidade disciplinar: Brasil 1890-193o, Rio de Janeiro, Paz e Terra, 1985.

RAGO, Margareth, Os prazeres da noite: prostituição e códigos da sexualidade feminina em São Paulo (1890-1930), São Paulo, Paz e Terra, 2008.

ROBERTS, Nickie, As prostitutas na História, Rio de Janeiro, Rosa dos Tempos, 1998. 


\section{* L'autore}

Débora Souza Cruz è studentessa del Programa de Pós-Graduação em História da Universidade Federal de Sergipe (UFS). Laureata in Storia presso l'Universidade Federal de Sergipe, ha ottenuto la specializzazione nell'insegnamento di Storia (Ensino de História: novas abordangens pela Faculdade São Luis de França). Ha partecipato per due anni al Programa de Educação Tutorial da UFS ed attualmente è membro del Grupo de Estudos do Tempo Presente e redattrice della rivista elettronica «Cadernos do Tempo Presente» (www.getempo.org).

URL: < http://studistorici.com/progett/autori/\#SouzaCruz >

\section{Per citare questo articolo:}

SOUZA CRUZ, Débora, «La costruzione dei discorsi sui cabaret e sulle prostitute durante l'epoca dell'Estado Novo ad Aracaju», Diacronie. Studi di Storia Contemporanea: Sulle tracce delle idee, 29/12/2012,

URL:< http://www.studistorici.com/2012/12/29/souza-cruz_numero_12/ >

Diacronie Studi di Storia Contemporanea $\}$ www.diacronie.it

Risorsa digitale indipendente a carattere storiografico. Uscita trimestrale. redazione.diacronie@hotmail.it

Comitato di redazione: Marco Abram - Jacopo Bassi - Luca Bufarale - Gianluca Canè - Alessandro Cattunar - Alice De Rensis - Barbara Galimberti - Deborah Paci - Fausto Pietrancosta - Matteo Tomasoni - Luca Zuccolo

Diritti: gli articoli di Diacronie. Studi di Storia Contemporanea sono pubblicati sotto licenza Creative Commons 2.5. Possono essere riprodotti a patto di non modificarne i contenuti e di non usarli per fini commerciali. La citazione di estratti è comunque sempre autorizzata, nei limiti previsti dalla legge. 